\title{
A GENERAL RUDIN-CARLESON THEOREM
}

\section{ERRETT BISHOP ${ }^{1}$}

1. Introduction. Rudin [7] and Carleson [4] have independently proved that if $S$ is a closed set of Lebesgue measure 0 on the unit circle

$$
L=\{z:|z|=1\}
$$

and if $f$ is a continuous function on $L$ then there exists a continuous function $F$ on

$$
D=\{z:|z| \leqq 1\}
$$

which is analytic on $D-L$ such that $F(z)=f(z)$ for all $z$ in $S$. It is the purpose of this paper to generalize this theorem. Before stating the generalization, we remark that the Rudin-Carleson theorem is closely related to a theorem of $F$. and M. Riesz, which states that any (finite, complex-valued, Baire) measure on $L$ which is orthogonal to all continuous functions $F$ on $D$ which are analytic on $D-L$ is absolutely continuous with respect to Lebesgue measure $d \theta$ on $L$. The proofs of the two theorems show that the results of RudinCarleson and of F. and M. Riesz are closely related. We shall state an abstract theorem which shows that the Rudin-Carleson theorem is a direct consequence of the F. and M. Riesz theorem. This abstract theorem will permit an automatic generalization of the RudinCarleson result to any situation to which the F. and M. Riesz result can be generalized. The theorem to be proved reads as follows:

THEOREM 1. Let $C(X)$ be the uniformly-normed Banach space of all continuous complex-valued functions on a compact Hausdorff space $X$. Let $B$ be a closed subspace of $C(X)$. Let $B^{\perp}$ consist of all (finite, complexvalued, Baire) measures $\mu$ on $X$ such that $\int f d \mu=0$ for all $f$ in $B$. Let $\hat{\mu}$ be the regular Borel extension of the Baire measure $\mu$. Let $S$ be a closed subset of $X$ with the property that $\hat{\mu}(T)=0$ for every Borel subset $T$ of $S$ and every $\mu$ in $B^{\perp}$. Let $f$ be a continuous complex-valued function on $S$ and $\Delta a$ positive function on $X$ such that $|f(x)|<\Delta(x)$ for all $x$ in $S$. Then there exists $F$ in $B$ with $|F(x)|<\Delta(x)$ for all $x$ in $X$ and $F(x)$ $=f(x)$ for all $x$ in $S$.

If $X$ is taken to be the set $L$ defined above and $B$ is taken to be those functions in $C(L)$ which are restrictions to $L$ of functions in

Received by the editors December 13, 1960.

1 This work was supported by the Sloan Foundation. 
$C(D)$ analytic on $D-L$, then by the F. and M. Riesz result any closed subset $S$ of $L$ of Lebesgue measure 0 satisfies the hypotheses of Theorem 1.

Therefore from Theorem 1 the Rudin-Carleson theorem follows.

By a general F. and M. Riesz theorem we mean a theorem to the effect that for certain closed subspaces $B$ of certain $C(X)$ there exists a non-negative measure $\mu_{0}$ on $X$ with respect to which all measures in $B^{\perp}$ are absolutely continuous. From Theorem 1 it follows that to each such general F. and M. Riesz theorem corresponds a general Rudin-Carleson theorem, which states that if $S$ is a closed subset of $X$ with $\hat{\mu}_{0}(S)=0$ then every continuous function on $S$ is the restriction to $S$ of some function in $B$.

There are various general F. and M. Riesz theorems which exist in the literature. We mention three of these.

(1) Bochner [3] and Helson and Lowdenslager [5] have proved an F. and M. Riesz theorem for $X=L \times L$, where $B$ is the subspace of $L \times L$ generated by all functions $z^{m} w^{n}$ with $(m, n)$ belonging to a sector of lattice points of opening greater than $\pi$. Here $\mu_{0}$ is Lebesgue measure on $L \times L$.

(2) Bishop $[1 ; 2]$ has proved a general F. and M. Riesz theorem for the boundary $X$ of a compact set $C$ in the complex plane whose complement is connected. Here $B$ consists of all continuous functions on $X$ which have extensions to $C$ analytic on $C-X$.

(3) Wermer [9] and Royden [6] (see also Rudin [8]) have proved a general F. and M. Riesz theorem for $X$ the boundary of a finite Riemann surface $R$. Here $B$ again is the set of continuous functions on $X$ which can be extended to be analytic on $R$ and continuous on $X \cup R$.

2. Proof of Theorem 1. We first prove a lemma from which the proof of Theorem 1 will be trivial.

Lemma. A ssume that $|f(x)|<r<1$ for all $x$ in $S$. Then under the hypotheses of Theorem 1 there exists $F$ in $B$ with $\|F\|<1$ and $F(x)$ $=f(x)$ for all $x$ in $S$.

Proof. Let $U_{r}$ be the set of all $g$ in $B$ of norm less than $r$. Let $\phi$ be the restriction mapping of $B$ into $C(S)$. The lemma states that $f \in \phi\left(U_{1}\right)$. We begin by showing that $f \in V_{r}$, where $V_{r}$ is the closure of $\phi\left(U_{r}\right)$. Assume otherwise. It follows from one of the many variants of the Hahn-Banach theorem that there exists a bounded linear functional $\alpha$ on $C(S)$ with $\alpha(f)>1$ and $|\alpha(h)|<1$ for all $h$ in $V_{r}$. By the Riesz representation theorem, there exists a measure $\mu_{1}$ on $S$ such that 


$$
\int g d \mu_{1}=\alpha(g)
$$

for all $g$ in $C(S)$. Define the linear functional $\beta$ on $B$ by $\beta(g)=\alpha(\phi(g))$. Since $\phi(g) \in V_{r}$ for all $g$ in $U_{r}$ it follows that $|\beta(g)|<1$ for all $g$ in $U_{r}$ so that $\|\beta\| \leqq r^{-1}$. By the Hahn-Banach theorem and the Riesz representation theorem it follows that there exists a measure $\mu_{2}$ on $X$ with $\left\|\mu_{2}\right\| \leqq r^{-1}$ and $\beta(g)=\int g d \mu_{2}$ for all $g$ in $B$. Thus the measure

$$
\mu=\mu_{1}-\mu_{2}
$$

is in $B^{\perp}$. Also

$$
\left|\int_{S} f d \mu\right| \geqq \int_{S} f d \mu_{1}-r\left\|\mu_{2}\right\|>1-r r^{-1}=0,
$$

contradicting the fact that $\hat{\mu}$ vanishes on all subsets of $S$. This contradiction shows that $f \in V_{r}$, so that there exists $F_{1}$ in $B$ with $\left\|F_{1}\right\|<r$ and $\left|f(x)-F_{1}(x)\right|<\lambda / 2$ for all $x$ in $S$, where $\lambda=1-r$. If we write $f_{1}(x)=f(x)-F_{1}(x)$, it follows by the result just proved, with $f$ replaced by $f_{1}$, that there exists $F_{2}$ in $B$ with $\left\|F_{2}\right\|<\lambda / 2$ and $\left|f_{1}(x)-F_{2}(x)\right|<\lambda / 4$ for all $x$ in $S$. Thus by induction we find a sequence $\left\{F_{n}\right\}$ of functions in $B$ with

$$
\left\|F_{n}\right\|<2^{-n+1} \lambda
$$

$n \geqq 2$,

and

$$
\left|f(x)-\sum_{k=1}^{n} F_{k}(x)\right|<2^{-n} \lambda, \quad n \geqq 2, x \in S .
$$

We define the function $F$ in $C(X)$ by

$$
F=\sum_{n=1}^{\infty} F_{n}
$$

Thus $F \in B$ and

$$
\|F\|<r+\sum_{n=2}^{\infty} 2^{-n+1} \lambda=r+\lambda=1 .
$$

Clearly also $F(x)=f(x)$ for all $x$ in $S$. This proves the lemma.

Proof of Theorem 1. Let $B_{0}$ be the closed subspace

$$
\{g: \Delta g \in B\}
$$

of $C(X)$. Then 


$$
\stackrel{\perp}{B_{0}}=\left\{\Delta \mu: \mu \in B^{\perp}\right\} \text {. }
$$

Thus $\nu(T)=0$ for all Borel sets $T \subset S$ and all $\nu$ in $B_{0}^{\perp}$. It follows from the lemma that there exists $F_{0}$ in $B_{0}$ with $\left\|F_{0}\right\|<1$ and $F_{0}(x)$ $=(\Delta(x))^{-1} f(x)$ for all $x$ in $S$. Let $F$ be the element $\Delta F_{0}$ of $B$. Thus $|F(x)|<|\Delta(x)|$ for all $x$ in $X$ and $F(x)=\Delta(x) F_{0}(x)=f(x)$ for all $x$ in $S$. This completes the proof.

\section{REFERENCES}

1. Errett Bishop, The structure of certain measures, Duke Math. J. vol. 25 (1958) pp. 283-290.

2. Boundary measures of analytic differentials, Duke Math. J. vol. 27 (1960) pp. 331-340.

3. Salomon Bochner, Boundary values of analytic functions in several variables and of almost periodic functions, Ann. of Math. vol. 45 (1944) pp. 708-722.

4. Lennart Carleson, Representations of continuous functions, Math. Z. vol. 66 (1957) pp. 447-451.

5. Henry Helson and David Lowdenslager, Prediction theory and Fourier series in several variables, Acta Math. vol. 99 (1958) pp. 165-202.

6. Halsey Royden, The boundary values of analytic and harmonic functions, O.O.R. Technical Report no. 19, DA-04-200-ORD-704, Stanford University.

7. Walter Rudin, Boundary values of continuous analytic functions, Proc. Amer. Math. Soc. vol. 7 (1956) pp. 808-811.

8. - Analyticity and the maximum modulus principle, Duke Math. J. vol. 20 (1953) pp. 449-458.

9. John Wermer, Subalgebras of the algebra of all complex-valued continuous functions on the circle, Amer. J. Math. vol. 78 (1956) pp. 225-242.

University of California, Berkeley 